\title{
¿Puede el aprendizaje de la geotecnia ser divertido?
}

\section{Julio Garzón-Roca ${ }^{\mathrm{a}}$, F. Javier Torrijo ${ }^{\mathrm{b}}$, Guillermo $\operatorname{Cobos}^{\mathrm{c}}$ y Luis Fernández ${ }^{\mathrm{d}}$}

${ }^{a}$ Depto. de Ingeniería del Terreno, ETSICCP, Universitat Politècnica de València, jugarro@upv.es

bopto. de Ingeniería del Terreno, ETSICCP, Universitat Politècnica de València, fratorec@trr.upv.es

${ }^{\mathrm{c}}$ Depto. de Ingeniería del Terreno, ETSICCP, Universitat Politècnica de València, gcobos@trr.upv.es

${ }^{\mathrm{d}}$ Depto. de Ingeniería del Terreno, ETSICCP, Universitat Politècnica de València, luiferp2@trr.upv.es

\begin{abstract}
Geotechnical engineering is usually an awkward topic for many students of civil engineering degrees who are often more focused on getting the final number of a given problem than in the process conducted to arrive to the solution. This issue is especially significant when teaching advance topics of geotechnical engineering such as Tunneling or Ground Improvement. Those topics are normally taught in the last years of the degrees and in many cases students are used to the classical teaching in Higher Education. All of this normally results in a low motivation of students, mainly interested in obtaining the degree itself, more than in learning. This article shows an active learning methodology based on autolearning which leads to involving the engineering students in those advanced geotechnical engineering topics, even enjoying with them. The core activity of the learning methodology is the preparation of a part of the subject by the own students, who work in groups and have to give a real lecture to their colleagues.
\end{abstract}

Keywords: Teaching innovation, Higher Education, active methodology, formative assessment, flips teaching

\section{Resumen}

La Geotecnia es en ocasiones una materia difícil e incómoda para el estudiante de ingeniería civil, el cual normalmente suelen centrarse más en obtener el número final de un problema determinado que en el proceso llevado a cabo para llegar a la solución. Esto es especialmente significativo cuando se enseñan temas avanzados de la disciplina, tales como el diseño y construcción de túneles o la mejora de terrenos, temas que se estudian en los últimos años de los grados, con unos alumnos acostumbrados a la enseñanza clásica en la Educación Superior. Todo esto lleva a que la motivación sea baja, estando los alumnos más interesados en obtener el título en sí, que en aprender. Así, este artículo muestra una metodología de aprendizaje activa basada en el autoaprendizaje que persigue involucrar a los alumnos en temas avanzados de la Geotecnia, llegando incluso a disfrutar con ellos. El núcleo central de la metodología es la preparación, en grupos, de una parte de la asignatura por parte de los propios alumnos, quienes deben dar una clase real a sus compañeros.

Palabras clave: Innovación docente, Educación Superior, metodología activa, aprendizaje por proyectos, clase inversa 


\section{Introducción}

La Geotecnia es a menudo una materia difícil e incluso incómoda para el alumno de Ingeniería Civil, normalmente más acostumbrado a enfocarse en obtener el número final del problema que en el proceso, usando el pensamiento ingenieril, llevado a cabo para plantear éste y llegar a una solución (que puede no ser única). Esto se hace especialmente significativo en los últimos años de los grados, cuando se enseñan temas avanzados de la Ingeniería Geotécnica, tales como el diseño y construcción de túneles o la mejora de terrenos. Los alumnos, acostumbrados a la enseñanza clásica de la Educación Superior, una educación muy reglada y basada fundamentalmente la transmisión de grandes conocimientos teóricos y técnicos, se encuentran así escasamente motivados, muchos de ellos deciden no asistir a las clases, y en el fondo están interesados principalmente en obtener el título en sí mismo, más que en el aprendizaje.

Se hace por tanto necesario desarrollar metrologías activas de forma que se consiga involucrar a los alumnos en el aprendizaje, considerando el pragmatismo inherente y típico de la profesión de ingeniero civil, y por qué no, consiguiendo que los alumnos lleguen incluso a disfrutar aprendiendo.

La asignatura objeto de la innovación que se presenta en esta comunicación pertenece al ámbito temático de la Ingeniería Geotécnica, se enmarca en el último año de grado y se imparte desde el año 2013, coincidiendo con la implantación de los grados y el Plan Bolonia, en la titulación de Ingeniero Civil.

Se tienen así fundamentalmente dos aspectos a trabajar:

- Implementar una evaluación continua, en contraposición a la fórmula tradicional de uno o dos únicos exámenes, la cual esté basada en un enfoque pragmático de la asignatura.

- Proponer actividades de carácter activo, que permita el seguimiento de la asignatura por parte de todo el alumnado, logre su motivación y los involucre en el aprendizaje de la asignatura y en el suyo propio.

\section{Objetivos}

La innovación planteada en esta comunicación se inscribe en el contexto de los alumnos de $4^{\circ}$ de Grado en Ingeniería Civil, los cuales se encuentran a las puertas de ejercer su titulación, y pretende conseguir una mayor motivación de éstos hacia la asignatura y la temática de ésta (la Ingeniería Geotécnica) al tiempo que proporcionales un cierto control sobre su propio aprendizaje.

A los alumnos se les ofrece la oportunidad de superar la asignatura mediante una evaluación continua a lo largo del cuatrimestre. Para ello, y como núcleo central de la innovación, los alumnos tienen que desarrollar por grupos una parte del temario de la asignatura, debiendo realizar entregas parciales (con retroalimentación posterior) al profesor encargado de tutorar al grupo así como una presentación final al resto de sus

(cc) BY-NC-ND 2018, Universitat Politècnica de València

Congreso IN-RED (2018) 
compañeros mediante la impartición de una clase real. Los temas desarrollados por los alumnos son eminentemente prácticos, y se les anima a que la presentación de éstos a sus compañeros sea lo más activa e interactiva posible, llegando incluso a lo lúdico, de forma que sea posible aprender divirtiéndose.

Se proponen así los siguientes objetivos a alcanzar con la innovación:

- Involucrar al alumno en la asignatura y despertar su interés por ella.

- Hacer partícipe al alumno de su propio aprendizaje y evaluación.

- Desarrollar la capacidad de autocrítica del alumno respecto a su propio conocimiento

- Fomentar el trabajo en grupo.

Esta innovación sigue el trabajo desarrollado por los profesores de la asignatura tanto fuera de la actividad docente como en varias experiencias docentes previas (Murillo, 2003; Zabala y Arnau, 2007; Santos et al., 2012; Torrijo et al., 2012, 2017), fomenta la motivación y la responsabilidad del alumno por su propio aprendizaje (Tedesco, 2010; Monroy et al., 2012) y combina fundamentalmente cuatro tipos de metodologías de aprendizaje: lección magistral participativa, aprendizaje cooperativo, aprendizaje autónomo y clase inversa (flipped classroom).

\section{Desarrollo de la innovación}

\subsection{Planteamiento}

La asignatura se divide fundamentalmente en dos partes, una primera más reglada y conducida por el profesorado, en la que se combina la lección magistral participativa y el aprendizaje cooperativo y autónomo, y una segunda parte, basada casi exclusivamente en un aprendizaje mediante clase inversa, en la que el alumno es el único protagonista del aprendizaje y en donde son los propios alumnos los que imparten (de manera grupal) las clases a sus compañeros.

La Tabla 1 recoge el calendario de la asignatura, relativo a las clases de teoría de aula y prácticas de aula, seguido durante el último curso académico (2017-2018), el cual, con pequeñas variaciones, es similar al planteado desde el inicio de la asignatura, en el año 2013. Además de estas clases de aula, en la asignatura también se realizan prácticas de laboratorio y una práctica de campo, en las que los alumnos son acompañados en el aprendizaje por uno o varios profesores. Este calendario es conocido por los alumnos desde el inicio de curso, de forma que además de la distribución del temario, los alumnos tienen conocimiento de la metodología docente y del profesor responsable de cada sesión.

Puesto que en general la terminología de aprendizaje es desconocida por el alumnado, se aprovecha el primer día de clase para explicar brevemente en qué consiste cada una de las metodologías que se emplearán a lo largo del curso, de forma que los alumnos tomen conciencia de lo que se espera de ellos en cada sesión y qué pueden esperar ellos del profesor, para que, en definitiva, adquieran control sobre su propio aprendizaje.

(cc) EY-NG-ND 2018, Universitat Politècnica de València

Congreso In-Red (2018) 
Tabla 1. Calendario de la asigntura (curso 2017-2018)

\begin{tabular}{|c|c|c|}
\hline Fecha & Tema & Metodología docente \\
\hline 05/09/2017 & Presentación de la asignatura & Lección magistral participativa \\
\hline 06/09/2017 & Planificación de reconocimientos del terreno & Aprendizaje autónomo \\
\hline $12 / 09 / 2017$ & Técnicas genéricas de prospección & Ap. cooperativo y autónomo \\
\hline $13 / 09 / 2017$ & Interpretación de datos geotécnicos en suelos y rocas & Aprendizaje autónomo \\
\hline $19 / 09 / 2017$ & Descripción de macizos rocosos & Lección magistral participativa \\
\hline 20/09/2017 & Instrumentación y auscultación geotécnica & Ap. cooperativo y autónomo \\
\hline $26 / 09 / 2017$ & Resistencia de macizos rocosos I & Lección magistral participativa \\
\hline $27 / 09 / 2017$ & Resistencia de macizos rocosos II & Lección magistral participativa \\
\hline 03/10/2017 & Deformabilidad de macizos rocosos & Lección magistral participativa \\
\hline 04/10/2017 & Casos prácticos de macizos rocosos & Ap. cooperativo y autónomo \\
\hline $10 / 10 / 2017$ & $\begin{array}{l}\text { Conferencia: Movimientos y deslizamientos del } \\
\text { terreno }\end{array}$ & Lección magistral participativa \\
\hline $11 / 10 / 2017$ & $\begin{array}{l}\text { Seminario abierto Cineforum (visionado de película- } \\
\text { documental) }\end{array}$ & Aprendizaje autónomo \\
\hline $17 / 10 / 2017$ & Estabilidad de taludes en rocas I & Ap. cooperativo y autónomo \\
\hline $18 / 10 / 2017$ & Estabilidad de taludes en rocas II & Ap. cooperativo y autónomo \\
\hline $24 / 10 / 2017$ & Estabilidad de taludes en suelos & Ap. cooperativo y autónomo \\
\hline $25 / 10 / 2017$ & $\begin{array}{l}\text { Técnicas de contención y corrección de } \\
\text { deslizamientos }\end{array}$ & Ap. cooperativo y autónomo \\
\hline $31 / 10 / 2017$ & Diseño y proyecto de la obra subterránea & Clase inversa \\
\hline 07/11/2017 & Cálculo geotécnico-estructural del túnel & Clase inversa \\
\hline 08/11/2017 & $\begin{array}{l}\text { Métodos de construcción de túneles y su } \\
\text { instrumentación y auscultación }\end{array}$ & Clase inversa \\
\hline $1411 / 2017$ & Casos prácticos sobre túneles & Clase inversa \\
\hline $15 / 11 / 2017$ & Compactación dinámica & Clase inversa \\
\hline $21 / 11 / 2017$ & Vibroflotación y columnas de grava & Clase inversa \\
\hline $22 / 11 / 2017$ & Precarga & Clase inversa \\
\hline $28 / 11 / 2017$ & Inyecciones y Jet Grouting & Clase inversa \\
\hline 29/11/2017 & Casos prácticos sobre mejora del terreno I & Clase inversa \\
\hline $05 / 12 / 2017$ & Casos prácticos sobre mejora del terreno II & Clase inversa \\
\hline $12 / 12 / 2017$ & Resumen y dudas de la asignatura I & Ap. cooperativo y autónomo \\
\hline $13 / 12 / 2017$ & Resumen y dudas de la asignatura II & Ap. cooperativo y autónomo \\
\hline
\end{tabular}

(cc) EY-NC-ND 2018, Universitat Politècnica de València 


\subsection{Visionado de la asignatura}

La primera parte de la asignatura es en cierto modo similar a las clásicas clases teóricas, de forma que es el profesor quien dirige las clases, estructura el contenido a tratar y fija en cierta medida el ritmo del aprendizaje. No obstante, se procura que el profesor no acapare todo el protagonismo. Así, en esta parte de la asignatura el profesor imparte unas pautas de puntos básicos de la materia que se va a trabajar y se proponen actividades a los alumnos. Se procura así que éstos tengan un papel lo más activo posible, desarrollando las clases de la forma más interactiva posible. Para ello, se emplean técnicas como el panel de expertos, el visionado de algunos fragmentos de videos documentales obtenidos directamente de internet (a través de la plataforma Youtube), el planteamiento de casos reales o la realización de sencillos tests empleando la aplicación informática kahoot. Con esto se pretende tanto motivar al alumno y captar su interés, como mostrarle diferentes alternativas a la típica lección magistral, metodología que es usualmente asociada por los alumnos con lo que es una clase.

La segunda parte de la asignatura se desarrolla con la metodología de clase inversa y su desarrollo se basa, como se ha indicado antes, en que son los propios alumnos los que imparten las clases. Mediante esta metodología de clase inversa se consigue dar el protagonismo al alumno así como la responsabilidad sobre su propio aprendizaje (Bergmann y Sams, 2012; Sams y Bergmann, 2013). El primer día de clase, tras informar a los alumnos sobre el funcionamiento del curso, la metodología a seguir y la evaluación, se les pide que formen de manera libre diez grupos de 3-5 personas (en función del número de alumnos), debiendo comunicar a los profesores en el plazo máximo de una semana qué personas forman cada uno de los grupos.

Aproximadamente dos semanas después de iniciado el curso, se cita a cada grupo para encomendarles la parte de la asignatura que deben preparar y sobre la que luego deben impartir una clase de 2 horas de duración. A cada grupo se le hace ver que van a ser ellos los responsables de una de las clases de la segunda parte de la asignatura, de forma que van a tomar el papel de profesor. Para preparar la clase, los alumnos cuentan con la ayuda de uno de los profesores de la asignatura, el cual tutora al grupo y controla el ritmo del trabajo a través de reuniones más o menos periódicas en las que se proporciona feedback a los alumnos, así como realizando sesiones de apoyo y acompañamiento. No obstante, el papel del profesor tutor es menor. Tras haber asignado a cada grupo el tema a impartir, para con ello centrarles en su desarrollo del aprendizaje, se indica a los alumnos que la preparación de la clase, tanto como quieren impartirla como los recursos a suministrar a sus alumnos (esto es, sus compañeros) es completamente responsabilidad suya. Esto crea en un primer momento desconcierto, miedo e incredulidad en los alumnos.

Superada esta primera etapa y una vez el grupo empieza a esbozar una primera idea de los contenidos de la clase, el tutor del grupo les recuerda la importancia que tienen los aspectos prácticos en la ingeniería civil y les anima a que la clase a impartir sea interactiva, dinámica y lúdica, de forma que se procure en lo posible que sus compañeros aprendan divirtiéndose. Esto en ocasiones lo resumimos con la siguiente frase: "Impartid la clase como si fuera la clase ideal que os hubiera gustado recibir en vuestra vida".

(cc) EY-NC-ND 2018, Universitat Politècnica de València 
Con ello se consigue que los alumnos preparen actividades realmente interesantes y divertidas. Así, en la Fig. 1 podemos ver a los alumnos del grupo responsable de impartir la clase realizando un kahoot a sus compañeros, en el que como premio se entregaba al ganador una tableta de chocolate.
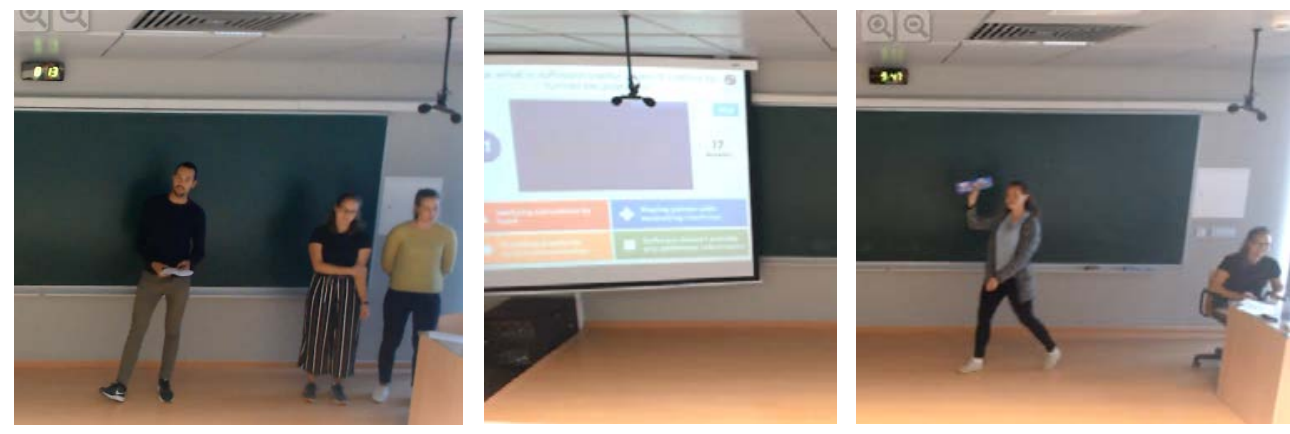

Fig. 1. Alumnos impartiendo el tema Cálculo geotécnico-estructural del túnel, correspondiente a la $18^{a}$ sesión de la asignatura

En la Fig. 2 podemos ver el aprendizaje de la compactación dinámica de suelos a través de una simulación real preparada por los alumnos del grupo responsable de impartir la clase con el fin de que sus compañeros entendiesen jugando los fundamentos de esta técnica y los efectos que lleva aparejada cuando es aplicada en un terreno.
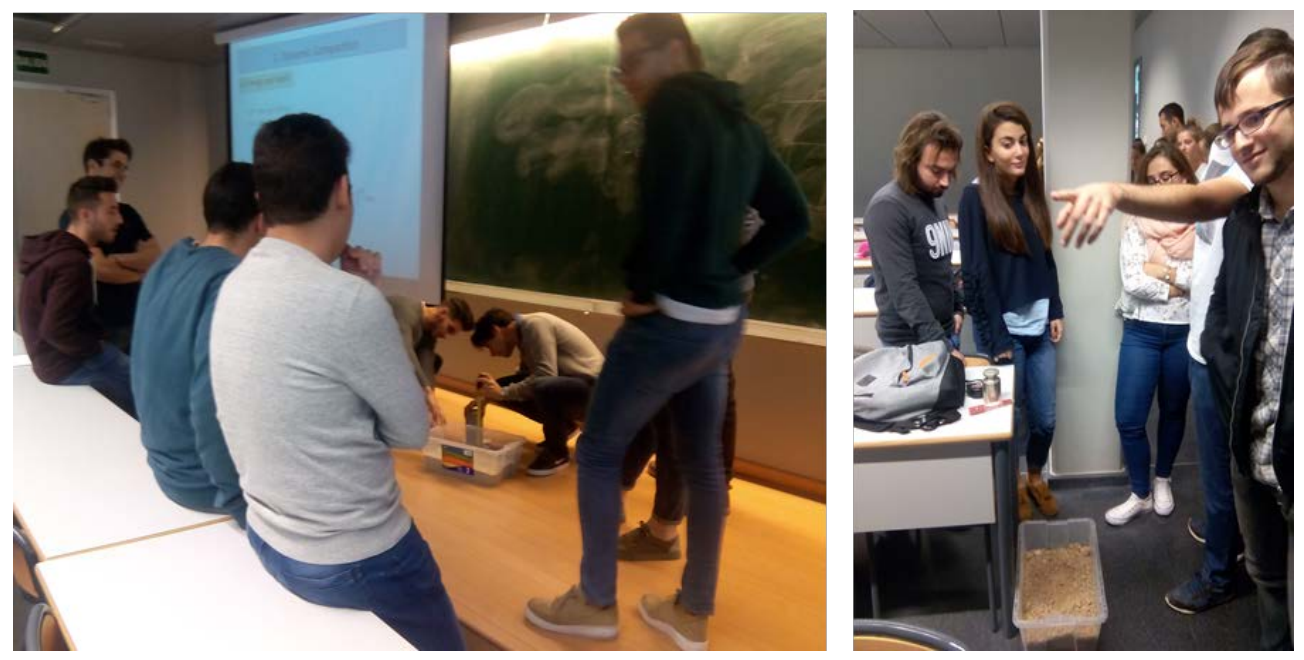

Fig. 2. Alumnos impartiendo el tema Compactación dinámica, correspondiente a la $21^{a}$ sesión de la asignatura

Este tipo de actividades, unido a la realidad de que son sus propios compañeros los que están impartiendo la clase (Fig. 3), lleva a que exista una participación notable por parte de los alumnos así como una implicación superior a la que se observaba antes de implantar esta metodología. Los alumnos terminan normalmente superando el miedo escénico a hablar en público y en algunas clases se producen debates sobre el tema impartido con una participación muy alta por parte de los alumnos.

Es interesante señalar que este cambio en el comportamiento de los alumnos se empieza a dar ya en la primera parte de la asignatura en la que las metodologías empleadas son la 
lección magistral participativa y el aprendizaje cooperativo y autónomo, ya que en ese momento ya llevan semanas trabajando para preparar lo que será su clase, lo que empieza a darles cierta seguridad en clase y los predispone a colaborar en la misma.
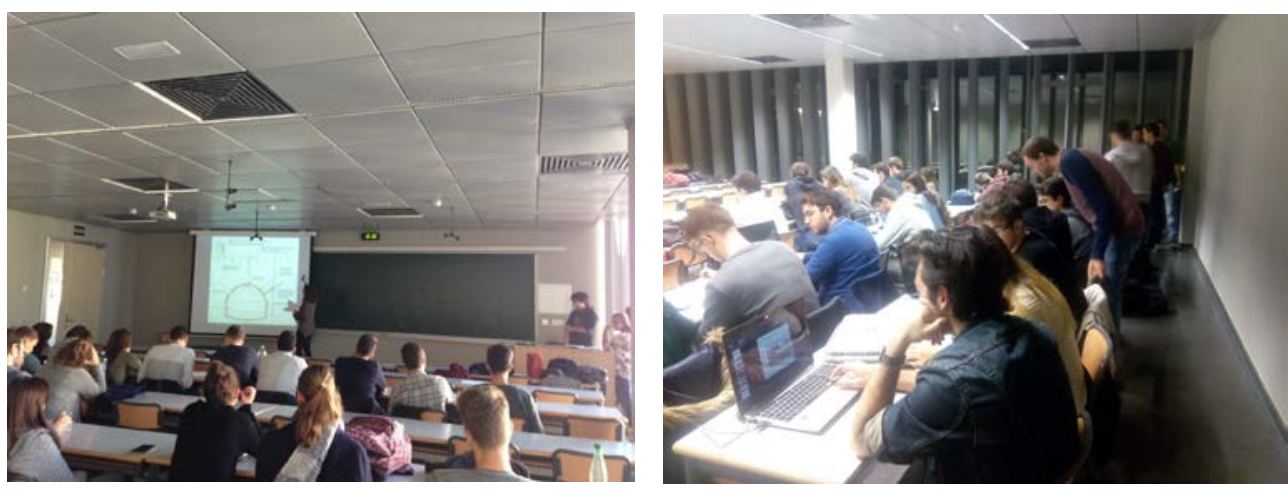

Fig. 3. Alumnos impartiendo los temas Métodos de construcción de túneles y su instrumentación y auscultación (izquierda) y Precarga (derecha) correspondiente a las $19^{a}$ y $23^{a}$ sesiones de la asignatura

\subsection{Recursos utilizados para el desarrollo de la asignatura}

Los recursos utilizados como material para el alumnado para lograr el éxito de la innovación pueden resumirse en:

- Información y datos necesarios para realizar su trabajo de forma autónoma y grupal: a los alumnos se les facilita al inicio del curso abundante material disponible en PoliformaT, en forma de material escrito y vídeos que sirven de punto de partida y acercamiento a la asignatura.

- Material teórico (apuntes y videos): se trata de un material que los alumnos pueden utilizar para retroalimentar su aprendizaje. Se encuentra disponible en PoliformaT, se comenta en clase y es la base para el desarrollo de las clases basadas en las metodologías aprendizaje autónomo y lección magistral participativa.

- Material teórico-práctico: este material puede ser utilizado por los alumnos para llevar a cabo de manera óptima el aprendizaje cooperativo y su trabajo grupal.

- Recursos bibliográficos: al principio del curso, así como durante la preparación de las clases por parte de los alumnos para ser impartidas durante la segunda parte de la asignatura, se facilita un amplio listado de referencias bibliográficas, tanto genéricas como específicas, que puedan consultar en los archivos de la Universidad.

\subsection{Evaluación}

Para los alumnos que siguen la metodología de la innovación planteada, la asignatura se evalúa en dos partes, correspondiendo a la división de la asignatura indicada anteriormente, a lo que se suma la evaluación de las prácticas laboratorio y de campo: 
- La primera parte de la asignatura, la cual se centra en aspectos más teóricos, se evalúa por medio de una prueba escrita de respuesta abierta, de carácter teóricopráctico, donde al alumno debe resolver cuestiones similares a las realizadas en clase. Esta prueba supone el 40\% de la calificación final.

- La segunda parte, correspondiente a las clases dadas por los alumnos, supone el $40 \%$ de la calificación final y se evalúa mediante coevaluación basada en tres ítems con el mismo peso (un tercio cada uno):

o Evaluación por pares, por parte de los compañeros presentes en la clase y ajenos al grupo que imparte la clase. La evaluación es anónima y se realiza una vez terminada la clase.

o Evaluación por pares, de cada uno de los alumnos del grupo que ha impartido la clase, de sus propios compañeros de grupo. La evaluación es anónima y se realiza una vez terminada la clase.

o Evaluación por parte del profesor que ha tutorizado al grupo tras comentar el trabajo desarrollado por los alumnos con el profesor o profesores presentes en la clase impartida por éstos (en el caso de que el profesor que tutoriza al grupo no esté presente).

- Las prácticas de laboratorio suponen un $10 \%$ de la calificación final y las evalúa el profesor responsable de las mismas en base a un trabajo escrito realizado individualmente por los alumnos.

- La práctica de campo suponen un $10 \%$ de la calificación final y la evalúa el profesor responsable de las misma en base a un trabajo escrito realizado individualmente por los alumnos.

Es interesante señalar que se da un peso importante a la evaluación por pares basada en la preparación de una parte del temario por parte de los alumnos y la impartición de una clase sobre dicho temario por ellos a sus compañeros, ya que se persigue fomentar la capacidad del alumno para seleccionar y presentar técnicamente la información que considere interesante para su público objetivo (en este caso, el resto de compañeros de clase), así como su capacidad para criticar técnicamente el trabajo de ingeniería realizado por sí mismo o por un tercero.

Asimismo, es importante destacar que al inicio del curso se plantea el sistema de evaluación de la asignatura, y a los alumnos se les da la oportunidad de ser evaluados tanto siguiendo la metodología anterior como a través de la fórmula tradicional consistente en dos exámenes. En este último caso, el primer examen es idéntico al examen de la primera parte de la innovación, mientras que el segundo examen es una prueba escrita de respuesta abierta, de carácter teórico-práctico, que versa sobre los temas tratados en la segunda parte de la asignatura.

\section{Resultados}

La Tabla 2 muestra los resultados de seguimiento de la asignatura por parte de los alumnos desde su implantación en el curso 2012-2013. Durante el primer año se seguía una 
metodología más tradicional, basada en casi exclusivamente el uso de la lección magistral y la evaluación del alumnado mediante dos exámenes. Ya en el curso 2013-2014 comenzó a implantarse la metodología expuesta en la presente comunicación, la cual ha sufrido poca variación, si bien sí se ha ido mejorando y puliendo poco a poco con el tiempo. Como puede observarse, el cambio de metodología supuso un claro y drástico descenso en el número de alumnos no presentados (quizás podría implicar más motivación), siendo menor a un $5 \%$.

Tabla 2. Resultados del seguimiento de la asignatura.

\begin{tabular}{lcccccc}
\hline & \multicolumn{6}{c}{ Curso } \\
\cline { 2 - 7 } & $\mathbf{2 0 1 3 - 2 0 1 4}$ & $\mathbf{2 0 1 3 - 2 0 1 4}$ & $\mathbf{2 0 1 4 - 2 0 1 5}$ & $\mathbf{2 0 1 5 - 2 0 1 6}$ & $\mathbf{2 0 1 6 - 2 0 1 7}$ & $\mathbf{2 0 1 7 - 2 0 1 8}$ \\
\hline No presentados & $32(27 \%)$ & $1(1 \%)$ & $5(3 \%)$ & $4(3 \%)$ & $4(5 \%)$ & $0(0 \%)$ \\
Presentados & $85(73 \%)$ & $84(99 \%)$ & $167(97 \%)$ & $131(97 \%)$ & $75(95 \%)$ & $86(100 \%)$ \\
\hline
\end{tabular}

En la Tabla 3 se recogen los resultados académicos de los alumnos presentados desde el curso 2012-2013 hasta el presente curso, 2017-2018. Se observa que la implementación de la innovación docente ha llevado aparejado un aumento general del porcentaje tanto de aprobados como de notas medias (Notables), si bien no se evidencia un aumento significativo en el número de Sobresaliente y Matrícula de Honor. Esto indica que la nueva metodología consigue mejorar el rendimiento académico de los alumnos medios, los cuales ven incrementadas sus calificaciones. Por su parte, los alumnos que ya eran excelentes siguen siéndolo.

Tabla 3. Resultados de las calificaciones de los alumnos presentados*

\begin{tabular}{lcccccc}
\hline & \multicolumn{7}{c}{ Curso } \\
\cline { 2 - 7 } & $\mathbf{2 0 1 2 - 2 0 1 3}$ & $\mathbf{2 0 1 3 - 2 0 1 4}$ & $\mathbf{2 0 1 4 - 2 0 1 5}$ & $\mathbf{2 0 1 5}-\mathbf{2 0 1 6}$ & $\mathbf{2 0 1 6 - 2 0 1 7}$ & $\mathbf{2 0 1 7 - 2 0 1 8}$ \\
\hline Suspensos & $12(14 \%)$ & $0(0 \%)$ & $0(0 \%)$ & $1(1 \%)$ & $0(0 \%)$ & $8(9 \%)$ \\
Aprobados & $65(77 \%)$ & $14(17 \%)$ & $46(27 \%)$ & $42(32 \%)$ & $33(44 \%)$ & $51(59 \%)$ \\
Notables & $7(8 \%)$ & $53(63 \%)$ & $119(68 \%)$ & $82(62 \%)$ & $39(52 \%)$ & $20(23 \%)$ \\
Sobresalientes & $1(1 \%)$ & $14(16 \%)$ & $2(1 \%)$ & $0(0 \%)$ & $1(1 \%)$ & $3(4 \%)$ \\
Mat. de Honor & $0(0 \%)$ & $3(4 \%)$ & $0(0 \%)$ & $6(5 \%)$ & $2(3 \%)$ & $4(5 \%)$ \\
Nota Media & 5,21 & 8,02 & 7,40 & 7,25 & 7,20 & 6,33 \\
Nota Máxima & 9,00 & 10,00 & 9,20 & 9,40 & 9,60 & 10,00 \\
Nota Mínima & 0,20 & 5,80 & 5,00 & 4,00 & 5,50 & 1,60 \\
Des. estándar & 5,87 & 0,96 & 1,22 & 1,25 & 0,80 & 1,65 \\
\hline \multirow{2}{*}{ Los por } & &
\end{tabular}

* Los porcentajes están respecto a los alumnos presentados

Es interesante señalar que del total de alumnos que han cursado la asignatura desde el curso 2013, momento en que se puso en marcha la innovación, de los 543 alumnos que decidieron seguir la asignatura de manera continua, casi la totalidad de estos han aprobado, tan sólo 9 han suspendido, varios de los cuales debido a un abandono de la asignatura. Asimismo, del 
análisis de las calificaciones se desprende que la media ha aumentado respecto al empleo de la metodología anterior entorno a punto y medio y la desviación estándar obtenida es reducida.

En lo que respecta a aspectos relacionados con el nivel de implicación, motivación y valoración de la asignatura por parte de los alumnos, la Tabla 4 recoge los resultados de una encuesta llevada a cabo por el ICE de la UPV en este último curso académico con el fin de evaluar la implantación de la metodología de la clase inversa. Debe señalarse que de los años anteriores no se tienen datos, por lo que no es posible realizar comparaciones. No obstante, desde un punto de vista cualitativo, se observó ya desde el curso 2013-2014 que la innovación planteada aumentaba significativamente el grado de implicación y la motivación de los alumnos.

Tabla 4. Resultados de la encuesta sobre la metodología aplicada, curso 2017-2018

\begin{tabular}{|c|c|c|c|c|c|}
\hline \multirow{2}{*}{ Pregunta } & \multicolumn{5}{|c|}{ Valoración* } \\
\hline & 1 & 2 & 3 & 4 & 5 \\
\hline \multicolumn{6}{|l|}{$\begin{array}{l}\text { Con la aplicación de la metodología realizada en esta } \\
\text { asignatura, muestra tu grado de acuerdo con las siguientes } \\
\text { afirmaciones }\end{array}$} \\
\hline He ido a clase con un trabajo previo realizado & $0 \%$ & $8 \%$ & $33 \%$ & $42 \%$ & $17 \%$ \\
\hline Ha contribuido a aumentar mi implicación en la asignatura & $8 \%$ & $8 \%$ & $17 \%$ & $42 \%$ & $25 \%$ \\
\hline Me ha resultado gratificante & $17 \%$ & $0 \%$ & $42 \%$ & $17 \%$ & $25 \%$ \\
\hline $\begin{array}{l}\text { Ha permitido que el profesor dispusiera de más tiempo en } \\
\text { clase para aclarar dudas }\end{array}$ & $8 \%$ & $33 \%$ & $25 \%$ & $17 \%$ & $17 \%$ \\
\hline Ha fomentado el trabajo en grupo & $0 \%$ & $17 \%$ & $8 \%$ & $42 \%$ & $33 \%$ \\
\hline Me ha ayudado a llegar mejor preparado a los exámenes & $25 \%$ & $8 \%$ & $17 \%$ & $50 \%$ & $0 \%$ \\
\hline \multicolumn{6}{|l|}{$\begin{array}{l}\text { Lo que más te ha gustado de la aplicación de la metodología } \\
\text { realizada en esta asignatura ha sido }\end{array}$} \\
\hline $\begin{array}{l}\text { Los materiales facilitados por el profesor (vídeos y } \\
\text { documentos utilizados) }\end{array}$ & $8 \%$ & $8 \%$ & $8 \%$ & $58 \%$ & $17 \%$ \\
\hline El trabajo realizado en clase (actividades de aula) & $0 \%$ & $17 \%$ & $33 \%$ & $33 \%$ & $17 \%$ \\
\hline El trabajo realizado en casa necesario para seguir las clases & $17 \%$ & $0 \%$ & $67 \%$ & $17 \%$ & $0 \%$ \\
\hline La relación con el profesor, más cercana y de trabajo conjunto & $0 \%$ & $8 \%$ & $8 \%$ & $33 \%$ & $50 \%$ \\
\hline La relación con mis compañeros & $8 \%$ & $0 \%$ & $17 \%$ & $50 \%$ & $25 \%$ \\
\hline El sistema de evaluación empleado & $8 \%$ & $17 \%$ & $0 \%$ & $42 \%$ & $33 \%$ \\
\hline \multicolumn{6}{|l|}{ Valoración general } \\
\hline Estoy muy satisfecho con esta experiencia & $8 \%$ & $17 \%$ & $25 \%$ & $42 \%$ & $8 \%$ \\
\hline Recomendaría esta experiencia a mis compañeros & $0 \%$ & $17 \%$ & $33 \%$ & $42 \%$ & $8 \%$ \\
\hline
\end{tabular}

(cc) EY-NC-ND 2018, Universitat Politècnica de València

Congreso IN-RED (2018) 
Del análisis de la Tabla 4 se deprende que la metodología activa planteada en la innovación logra los objetivos de fomentar el trabajo en grupo y de aumentar el nivel de implicación en la asignatura. Esto último parece que tiene un doble efecto colateral, pues motiva a que el alumno acuda a clase con el trabajo previo (ver un video, resolver un ejercicio práctico, leer algún fragmento de un tema, etc.) realizado y ayuda en cierta medida al alumno a llegar mejor preparado a los exámenes.

Por otra parte, lo que más valoran los alumnos, además del material facilitado por los profesores, es la posibilidad de aprender junto con sus compañeros tanto dentro como fuera del aula. Como era de esperar, la creación de un buen entorno social favorece el aprendizaje y esto queda reflejado en la experiencia de los alumnos con la asignatura. El sistema de evaluación empleado también es positivamente valorado por los alumnos, los cuales aprecian el ofrecimiento que les da la asignatura de ser aprobada mediante una evaluación continua y no a través de uno o dos exámenes independientes del curso en sí.

Finalmente, es importante señalar que a la gran mayoría de los alumnos les ha gustado la metodología docente seguida y recomendarían a sus compañeros seguirla en los cursos venideros.

\section{Conclusiones}

Tras cinco cursos aplicando la nueva metodología activa basada en el autoaprendizaje y el empleo de la clase inversa, es indudable que su implantación ha conseguido aumentar la motivación de los alumnos así como su predisposición para participar activamente en la asignatura.

La innovación ha permitido que los alumnos transformen, al menos en cierta medida, su típica visión de memorizar extrañas teorías y sistematizar la resolución de los problemas del examen, enfoque clásico de los alumnos de Ingeniería Civil ante una materia teóricopráctica como la Geotecnia, a un nuevo marco en el que son ellos los que aprenden de forma continua, día a día, acompañados de los profesores que les ayudan a afrontar los obstáculos. Los alumnos son los verdaderos protagonistas de las clases, llegando incluso a ser ellos quienes las imparten al resto de compañeros. Y todo ello bajo un enfoque lo más práctico e interactivo posible, e incluso lúdico en algunos casos, que busca claramente que el alumno aprenda haciendo así como que aprenda divirtiéndose.

Así, la metodología educativa implantada ha supuesto un avance en la evaluación formativa completa de los alumnos, ha mejorado su rendimiento académico, en especial el de los alumnos medios, y ha reducido ostensiblemente el absentismo académico así como el fracaso escolar en la Educación Superior, teniéndose unas cifras de alumnos presentados cercanas todos los años al 100\%, así como una tasa de alumnos suspensos muy reducida o incluso nula.

Finalmente, es interesante destacar que la innovación ha sido el fruto del trabajo cooperativo de todos los profesores de la asignatura, quienes han colaborado en mayor o en menor medida a que la innovación haya sido implantada exitosamente a lo largo de estos cinco cursos académicos. El trabajo en equipo y la reflexión compartida entre nosotros, tal 
y como indicaban Santos et al. (2012), ha permitido salvar las inseguridades generadas por hacer algo diferente e innovador.

\section{Referencias}

BERGMANN, J. y SAMS, A. (2012). "Flip your classroom : Talk to Every Class Every Day”. ISTE, Washington D.C.

KAHOOT. Kahoot! < https://kahoot.com /> [Consulta: 15 de marzo de 2017]

MONROY, R., TORRIJO, F.J. y HERNÁNDEZ-PINA, F. (2012). "Lecturers' perceptions of students' learning needs in geo-engineering in Spain” en McCabe, Pantazidou \& Phillips. Shaking the Foundations of Geo-engineering Education. London: Taylor \& Francis Group. 225-230.

MURILLO, F.J. (2003). "El movimiento teórico-práctico de Mejora de la Escuela. Algunas lecciones aprendidas para transformar los centro docentes” en Revista Electrónica Iberoamericana sobre Calidad, Eficacia y Cambio en Educación, vol. 1, issue 2, p. 1-22.

SAMS, A. y BERGMANN, J. (2013). Flip Your Students’ Learning” en Technology-Rich Learning, $70, n^{\circ} 6$.

SANTOS, M., CASTEJÓN, F.J. y MARTÍNEZ, L.F. (2012). "La innovación docente en evaluación formativa y metodología participativa. Un proyecto compartido a raíz de la implantación de los nuevos grados” en Psychology, Society \& Education, vol. 4, issue 1 p. 73-86.

TEDESCO, J.C. (2010). “La educación en el horizonte 2020”. Fundación Santillana, Madrid.

TORRIJO, F.J., CORTÉS, R. y VALIENTE, R. (2012). “Indagación y mejora docente en el campo de la Geología Aplicada”. Universidad Politécnica de Valencia. En Jornadas de Innovación Eductaiva 2012. Valencia. 376-378.

TORRIJO, F.J., GARZÓN-ROCA, J., COBOS, G. y ALIJA, S. (2017). “Implementación de la metodología de Clase Inversa en el campo de la Ingenieria del Terreno”. International Conference on Innovation, Documentation and Education, INNODOCT 2017, 25 - 27 de Octubre de 2017, Valencia.

ZABALA, A. y ARNAU, L. (2017). “11 ideas clave. Cómo aprender y enseñar competencias”. Colección Ideas Clave. Serie Didáctica / Diseño y desarrollo curricular. Editorial Graó de Irif, S.L., Barcelona. 